\title{
Determining Total Phenolic Compound, Tannin and Anthocyanin Ratios of Grape Varieties Cultivated in Şanlıurfa Province
}

\author{
Aslı POLAT ${ }^{1 *}(\mathbb{C})$, İsmail RASTGELDi ${ }^{2}$ (i) , Sadettin GÜRSÖZ $Z^{3}$ \\ ${ }^{1}$ Tekirdağ Viticulture Research Institute, Tekirdağ, Turkey \\ ${ }^{2}$ GAP Agriculture Research Institute, Şanlıurfa, Turkey \\ ${ }^{3}$ Harran University, Agriculture Faculty, Horticulture Department, Şanlıurfa, Turkey
}

How to cite: Polat, A., Rastgeldi, I., \& Gürsöz, S. (2022). Determining total phenolic compound, tannin and anthocyanin ratios of grape varieties cultivated in Şanlıurfa province. Viticulture Studies (VIS), 2(1), $01-12$. https://doi.org/10.52001/vis.2021.6.01.12

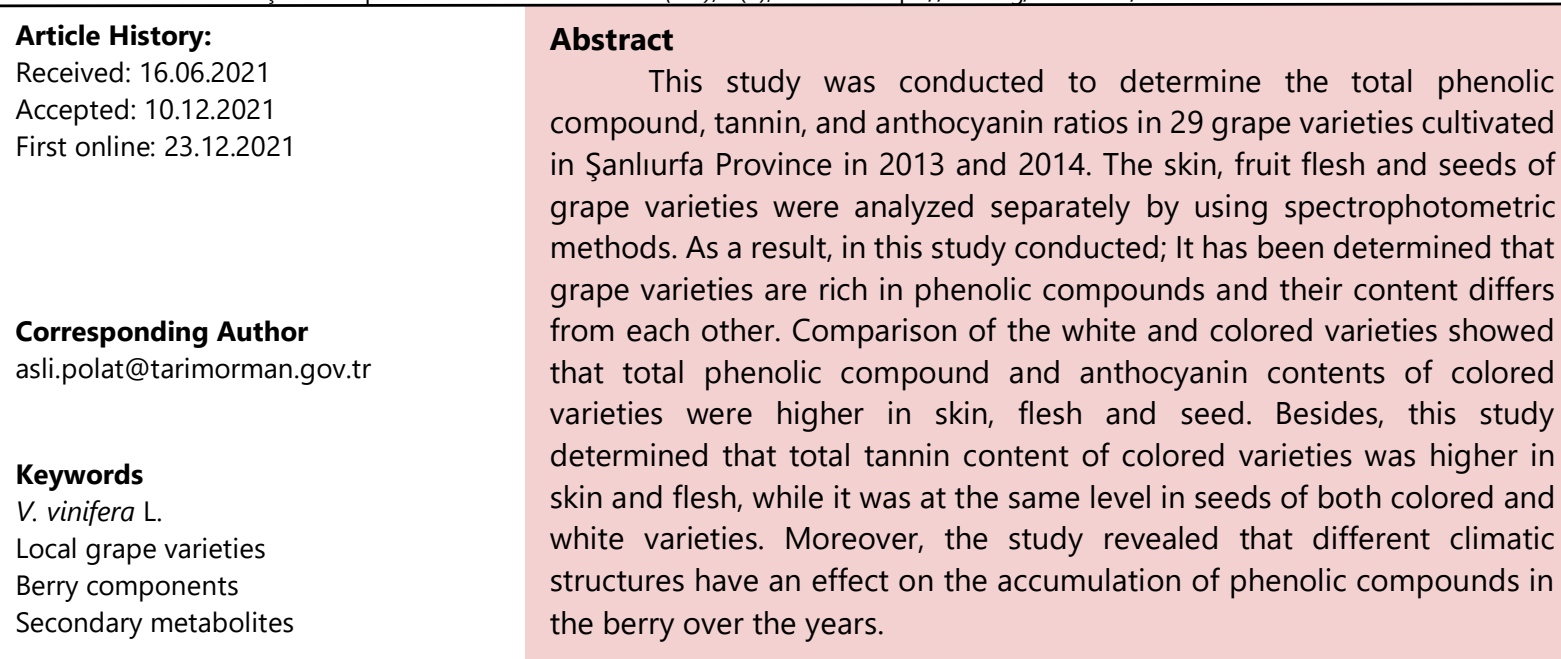

\section{Introduction}

The role of grape in nutrition has been known since ancient times and it still preserves this feature today. Factors such as variety, ecological conditions and cultivation techniques are effective on the composition of the grape (Yavaş and Fidan, 1986).

Determining the chemical composition of grape and grape products, which have important roles in human nutrition and health, is considered to play an important role in the formation of a more conscious consumption habit, as it enables a selection according to the purpose and need. In addition, it is particularly important in terms of revealing the values of table grapes, dried and wine grapes of local varieties (Aras, 2006).

Currently, phenolic compounds, which are the subject of studies in many fields such as agriculture, food, biology, chemistry and pharmacology are synthesized not only during the normal development of plants; but also, in cases such as being infected, injured and exposed to UV light (Naczk and Shahidi, 2004).

They are considered as secondary plant metabolites, knowing that they are formed as side compounds during aromatic amino acid metabolism (Van Buren, 1970; Acar, 1998; Naczk and Shahidi, 2004). Secondary plant metabolites are compounds that are not vital for the whole or certain parts of the plant. These compounds are classified into a group of characteristic features that include biosynthetic precursors (phosphoenolpyruvate, pyruvate, acetate and some amino acids, acetyl CoA and malonyl CoA), including structural diversity and synthesis. (Robards et al., 1999).

Grape is a plant species rich in phenols and phenols are mainly found in the skin, stem, leaves and seeds of the grape (Pastrana-Bonilla et al., 2003; Makris et al., 2008). The phenolic content of grapes differs depending on the variety, soil structure, climate, growing conditions and if it is contaminated by fungal diseases (Bruno and Sparapano, 2007). 
The most widely studied metabolites in viticulture are phenolic compounds. It has been reported that these substances are found in different forms and in different amounts in all organs of the vine such as roots, branches, shoots, leaves, bunches and berries (Sivritepe, 2001).

The most important group of phenolic compounds found in grapes are anthocyanins which are responsible for the color (Cemeroglu et al., 2001). Anthocyanins are natural color pigments that give their distinctive red, blue and purple hues (Costa et al., 2000; Ho et al., 2001; Camire et al., 2002).

The anthocyanin content of grapes is an important quality criterion for winemaking. Tannins, another group of phenolic compounds, are responsible for the formation of taste in grapes and are predominantly found in the pedicel, skin and seeds. In general, the amount of tannins in the berry reaches the highest level just before the veraison phase, and decreases towards maturity (Harborne and Grayer 1993).

Previous findings obtained by authors have revealed that some of the varieties identified in the region by the study of Gürsöz (1993) could not survive until today, while some of them were in danger of extinction. It is necessary to take some precautions and perform certain studies to bring these varieties back to production. Determining the characteristic features of the varieties known only in the region is the main work to start with.

\section{Material and Methods}

This study was conducted by observing and taking samples from the 29 grape varieties determined in different districts and parcels in Şanlıurfa province in 2013 and 2014. The grape variety from each district is as follows: from Akçakale district; Tahannebi, Perlette, Yediveren (table grapes) Öküzgözü, Syrah, Chardonnay, Cabernet Sauvignon (wine grapes), from Bozova district; Hatun Parmağı, Şeffafi (table grapes), Tilgören, Azezi (must and wine grapes), from Hilvan District; Horoz Karası, Kabarcık, Top Üzüm, Hönüsü, Su Üzümü, Serpenekıran (table grapes), from Karaköprü District; Küllahi, Zeyni, Çiloreş, Kara Kabarcık (table grape), Çilorut (must and table grape), Sergi Karası (Dried), from Siverek District; Simore, Elma Üzümü, Kızıl Banki,
Şire, Hasani, Ağ Banki (Table grapes). The soils are generally basic in character, rich in lime and poor in organic matter.

For phenolic compound, tannin and anthocyanin analysis, the skin, flesh and seed parts of the berries were separated and analyzed separately. Analyzes were performed as 3 replications.

\section{Determination of Total Phenolic Compounds and Tannin}

Extraction: $5 \mathrm{~g}$ of skin, flesh or seed samples were weighed and cut into small pieces by using a scalpel. Later, $50 \%$ ethanol was added to the samples and they were thoroughly crushed in the homogenizer. Then, they were filtered through whatmann paper and the $50 \%$ ethanol was added to a final volume of $25 \mathrm{~mL}$. They were kept in $4{ }^{\circ} \mathrm{C}$ until the anlysis is done (Yu and Meredith, 1986; Tangolar et al., 1999).

Determination of total phenolic compound: Analysis of total phenolic compounds was determined using Folin-Ciocalteu colorimetric method defined by Singleton and Rossi (1965), and the results were noted in gallic acid ( $\mathrm{mg} \mathrm{kg}$ $\left.{ }^{1}\right)$.

Determination of total tannins: The prepared extractions were analyzed according to AOAC, (1998).

\section{Determination of Total Anthocyanin Content}

Extraction: After separating the skin, fruit flesh and seeds of 10 randomly selected grape berries and recording their weight, they were kept separately at $30{ }^{\circ} \mathrm{C}$ in $40 \mathrm{~mL}$ solution with a $\mathrm{pH}$ of 3.2 for 72 hours. Then they were stored at $-20{ }^{\circ} \mathrm{C}$ until analysis (Di Stefano and Cravero, 1991).

Determination of Total Anthocyanin: The samples were analyzed on the spectrophotometer after diluting them in $1 / 10$ hydrochloric ethanol [EtOH (95\%): $\mathrm{H}_{2} \mathrm{O}$ (deionized distilled water): $\mathrm{HCl}$ (70: 30: $1 \mathrm{v} / \mathrm{v} / \mathrm{v})$ ]. The total anthocyanin amounts were determined according to Di Stefano and Cravero, (1991) by calculating the absorbance values obtained expressed in equivalents of malvidin 3-glikozide compound $\left(\mathrm{mg} \mathrm{kg}^{-1}\right)$.

Hach lange DR 5000 UV-VIS model spectrophotometer was used for analyzes. 


\section{Statistical Analysis}

After all the data obtained were divided into two as colored and white grapes, they were evaluated statistically according to the randomized blocks trial design. The results were evaluated by analysis of variance, and the differences between years and varieties were revealed by Duncan multiple comparison test. For this purpose, "SPSS 16" software was used.

\section{Results and Discussion}

Maturity criteria of grape varieties harvested in 2013 and 2014 are given in Table 1.

\section{Total Phenolic Compound Content}

It was found that total phenol content in the skin of the white grape varieties as the average of both years was $167 \mathrm{mg} \mathrm{kg}^{-1}$ in the Kabarcık and it was the lowest value, in Chardonnay it was $504 \mathrm{mg} \mathrm{kg}^{-1}$ as the highest value. Total phenolic content of fruit flesh was found as $51 \mathrm{mg} \mathrm{kg}^{-1}$ in Ağ Banki is the lowest and $178 \mathrm{mg} \mathrm{kg}^{-1}$ in Tahannebi as the highest. Besides that, it was determined that the lowest total phenol content in the seed was $364 \mathrm{mg} \mathrm{kg}^{-1}$ in the Ağ Banki and the highest was $731 \mathrm{mg} \mathrm{kg}^{-1}$ in the Çiloreş (Table 2). Among the colored varieties as the average of the years, total phenol content in the skin of the fruit was found to be lowest in Kızıl Banki with the value of $366 \mathrm{mg} \mathrm{kg}^{-1}$ and it was determined as the highest with the value of $511 \mathrm{mg} \mathrm{kg}^{-1}$ in Kara Kabarcık. Fruit flesh was found to have the lowest total phenol content and it was $61 \mathrm{mg} \mathrm{kg}^{-1}$ in Yediveren, while by 172 $\mathrm{mg} \mathrm{kg}^{-1}$ it was the highest in Horoz Karası.

Table 1. Maturity criteria of grape varieties harvested

\begin{tabular}{|c|c|c|c|c|c|c|c|c|c|c|}
\hline \multirow[t]{2}{*}{ Varieties } & \multicolumn{2}{|c|}{ Harvest date } & \multicolumn{2}{|c|}{ Brix (\%) } & \multicolumn{2}{|c|}{ pH } & \multicolumn{2}{|c|}{ Acidity (\%) } & \multicolumn{2}{|c|}{$\begin{array}{c}\text { Maturity } \\
\text { Index }\end{array}$} \\
\hline & 2013 & 2014 & 2013 & 2014 & 2013 & 2014 & 2013 & 2014 & 2013 & 2014 \\
\hline Ağ Banki & Sep, 5 & Sep, 11 & 23.30 & 19.50 & 3.99 & 3.73 & 0.448 & 0.408 & 52.10 & 47.80 \\
\hline Azezi & Sep, 9 & Sep, 4 & 18.60 & 18.50 & 3.72 & 3.99 & 0.296 & 0.327 & 63.00 & 56.40 \\
\hline Chardonnay & Aug, 27 & Agu, 11 & 20.70 & 21.20 & 3.74 & 3.60 & 0.621 & 0.572 & 33.30 & 37.20 \\
\hline Çiloreş & Sep, 1 & Agu, 29 & 21.20 & 19.00 & 3.74 & 3.82 & 0.330 & 0.369 & 64.20 & 51.60 \\
\hline Çilorut & Aug, 21 & Agu, 20 & 17.00 & 18.20 & 3.80 & 3.86 & 0.430 & 0.464 & 39.50 & 39.10 \\
\hline Elma Üzümü & Sep, 5 & Agu, 26 & 14.60 & 20.00 & 3.49 & 3.76 & 0.503 & 0.532 & 29.00 & 37.50 \\
\hline Hasani & Aug, 26 & Sep, 11 & 16.50 & 18.10 & 3.52 & 3.78 & 0.610 & 0.492 & 27.10 & 36.80 \\
\hline Hatun Parmağı & Aug, 12 & Agu, 7 & 17.30 & 17.00 & 3.98 & 3.39 & 0.475 & 0.423 & 36.40 & 40.20 \\
\hline Kabarcık & Aug, 15 & Agu, 18 & 16.10 & 17.70 & 3.83 & 3.85 & 0.473 & 0.421 & 34.10 & 42.00 \\
\hline Küllahi & Aug, 12 & Agu, 12 & 15.50 & 18.30 & 3.83 & 3.84 & 0.480 & 0.277 & 32.20 & 66.20 \\
\hline Perlette & July, 29 & July, 24 & 20.30 & 19.10 & 3.68 & 3.59 & 0.505 & 0.669 & 40.20 & 28.50 \\
\hline Serpenekıran & Sep, 9 & Sep, 8 & 19.30 & 18.50 & 3.76 & 3.77 & 0.399 & 0.367 & 48.40 & 50.40 \\
\hline Simore & Aug, 26 & Agu, 26 & 19.90 & 19.80 & 3.32 & 3.56 & 0.767 & 0.582 & 26.00 & 34.00 \\
\hline Su Üzümü & Sep, 9 & Sep, 3 & 20.70 & 17.30 & 3.94 & 4.01 & 0.492 & 0.347 & 42.00 & 49.80 \\
\hline Şeffafi & Aug, 12 & Agu, 7 & 19.40 & 19.70 & 3.84 & 3.27 & 0.367 & 0.449 & 52.90 & 43.80 \\
\hline Şire & Sep, 12 & Sep, 11 & 19.20 & 18.20 & 3.39 & 3.65 & 0.416 & 0.478 & 46.30 & 38.00 \\
\hline Tahannebi & July, 15 & July, 22 & 17.50 & 23.00 & 4.00 & 4.21 & 0.389 & 0.451 & 45.00 & 50.90 \\
\hline Zeyni & Aug, 12 & Agu, 7 & 15.90 & 20.10 & 3.88 & 3.85 & 0.448 & 0.462 & 35.40 & 43.60 \\
\hline Cabernet Sauvignon & Aug, 27 & Agu, 25 & 20.80 & 20.10 & 3.82 & 3.71 & 0.588 & 0.643 & 35.40 & 31.30 \\
\hline Horoz Karası & Aug, 15 & Agu, 18 & 18.10 & 19.50 & 3.95 & 3.97 & 0.482 & 0.536 & 37.60 & 36.40 \\
\hline Hönüsü & Sep, 9 & Agu, 29 & 18.10 & 19.40 & 3.41 & 3.63 & 0.406 & 0.534 & 44.70 & 36.30 \\
\hline Kara Kabarcık & Aug, 26 & Sep, 3 & 13.30 & 17.20 & 3.87 & 4.34 & 0.381 & 0.267 & 34.90 & 64.50 \\
\hline Kızıl Banki & Sep, 5 & Sep, 3 & 16.20 & 16.70 & 3.88 & 4.28 & 0.277 & 0.361 & 58.60 & 46.30 \\
\hline Öküzgözü & Aug, 17 & Agu, 11 & 19.40 & 17.20 & 4.13 & 3.82 & 0.350 & 0.562 & 55.50 & 30.70 \\
\hline Sergi Karası & Aug, 21 & Agu, 20 & 24.10 & 24.20 & 3.98 & 3.86 & 0.506 & 0.471 & 47.70 & 51.30 \\
\hline Şiraz & Aug, 27 & Agu, 25 & 24.70 & 24.10 & 3.96 & 4.05 & 0.472 & 0.578 & 52.20 & 41.70 \\
\hline Tilgören & Aug, 12 & Agu, 18 & 14.40 & 18.70 & 3.65 & 3.89 & 0.577 & 0.417 & 25.00 & 44.80 \\
\hline Top Üzüm & Sep, 9 & Agu, 29 & 16.20 & 18.70 & 3.83 & 3.30 & 0.485 & 0.461 & 33.40 & 40.60 \\
\hline Yediveren & Aug, 17 & Agu, 21 & 17.70 & 20.20 & 4.13 & 4.23 & 0.434 & 0.350 & 40.80 & 57.70 \\
\hline
\end{tabular}


Hönüsü was determined to have the lowest total phenol content of the seed with $513 \mathrm{mg} \mathrm{kg}^{-1}$ and the highest value was found to be $1183 \mathrm{mg} \mathrm{kg}^{-1}$ in the Shiraz (Table 3).

The amount of total phenolics in grapes varies according to the year and variety and decreases during the ripening period (Doshi et al., 2006; Jin et al., 2009). Similarly, in a similar study carried out under the conditions of Tekirdağ Province in 2007 and 2008, it has been reported that the total amount of phenolic compounds in grapes varies according to the

Table 2. Total phenolic compound contents of white grapes

\begin{tabular}{|c|c|c|c|c|c|c|}
\hline \multirow{3}{*}{ Varieties } & \multicolumn{6}{|c|}{ Total Phenolic Compound (mg kg-1) } \\
\hline & \multicolumn{3}{|c|}{2013} & \multicolumn{3}{|c|}{2014} \\
\hline & Skin & Flesh & Seed & Skin & Flesh & Seed \\
\hline Ağ Banki & $410 \pm 1.14 f$ & $47 \pm 1.07 j$ & $365 \pm 4.031$ & $432 \pm 0.64 d$ & $56 \pm 1.52 i$ & $363 \pm 1.87 \mathrm{~g}$ \\
\hline Azezi & $265 \pm 2.12 j$ & $164 \pm 2.00 \mathrm{~b}$ & $643 \pm 4.08 b$ & $176 \pm 1.60 \mathrm{j}$ & $126 \pm 1.72 c$ & $570 \pm 4.20 \mathrm{ef}$ \\
\hline Chardonnay & $487 \pm 2.31 \mathrm{a}$ & $38 \pm 0.59 \mathrm{k}$ & $580 \pm 5.11$ ef & $370 \pm 3.73 e$ & $74 \pm 0.51 \mathrm{~h}$ & $684 \pm 3.40 b$ \\
\hline Çiloreş & $375 \pm 2.55 h$ & $35 \pm 1.32 \mathrm{k}$ & $675 \pm 6.12 a$ & $437 \pm 3.07 \mathrm{~cd}$ & $73 \pm 0.79 h$ & $787 \pm 4.45 a$ \\
\hline Çilorut & $112 \pm 0.83$ no & $47 \pm 1.10 \mathrm{j}$ & $535 \pm 4.91 \mathrm{~h}$ & $368 \pm 3.20 \mathrm{ef}$ & $95 \pm 0.72 \mathrm{ef}$ & $560 \pm 5.10$ efg \\
\hline Elma Üzümü & $136 \pm 2.47 \mathrm{~m}$ & $71 \pm 1.24 \mathrm{~h}$ & $628 \pm 5.09 c$ & $227 \pm 2.49 \mathrm{i}$ & $73 \pm 0.40 \mathrm{~h}$ & $592 \pm 2.73 \mathrm{de}$ \\
\hline Hasani & $262 \pm 0.40 \mathrm{j}$ & $54 \pm 1.62 i$ & $514 \pm 3.45 i$ & $361 \pm 3.26 f$ & $55 \pm 0.85 i$ & $564 \pm 0.52$ efg \\
\hline Hatun Parmağı & $254 \pm 1.78 \mathrm{k}$ & $54 \pm 1.35 i$ & $567 \pm 4.97 \mathrm{fg}$ & $375 \pm 1.83 e$ & $93 \pm 1.08$ ef & $551 \pm 2.44 \mathrm{fg}$ \\
\hline Kabarcık & $108 \pm 0.04 \circ$ & $96 \pm 1.20 \mathrm{~g}$ & $501 \pm 4.81 i$ & $225 \pm 2.08 \mathrm{i}$ & $99 \pm 1.35 \mathrm{e}$ & $582 \pm 0.49 e$ \\
\hline Küllahi & $116 \pm 2.57 n$ & $118 \pm 1.98 \mathrm{e}$ & $485 \pm 3.87 j$ & $287 \pm 2.98 \mathrm{~h}$ & $101 \pm 1.27 e$ & $558 \pm 2.58 \mathrm{efg}$ \\
\hline Perlette & $418 \pm 1.32 \mathrm{e}$ & $123 \pm 2.51 \mathrm{e}$ & Seedless & $469 \pm 1.68 b$ & $147 \pm 2.68 \mathrm{~b}$ & Seedless \\
\hline Serpenekıran & $132 \pm 0.54 \mathrm{~m}$ & $129 \pm 3.40 d$ & $587 \pm 5.08 \mathrm{de}$ & $232 \pm 2.05 i$ & $115 \pm 1.27 d$ & $634 \pm 1.93 d$ \\
\hline Simore & $445 \pm 2.36 d$ & $105 \pm 1.30 f$ & $571 \pm 1.40$ efg & $361 \pm 2.54 f$ & $105 \pm 1.38 \mathrm{e}$ & $591 \pm 2.79 \mathrm{de}$ \\
\hline Su Üzümü & $384 \pm 5.03 \mathrm{~g}$ & $105 \pm 1.49 f$ & $584 \pm 7.79 \mathrm{de}$ & $340 \pm 1.06 \mathrm{~g}$ & $108 \pm 0.77 e$ & $667 \pm 3.52 c$ \\
\hline Şeffafi & $234 \pm 1.14 \mid$ & $107 \pm 1.49 f$ & $455 \pm 3.10 k$ & $281 \pm 2.50 h$ & $85 \pm 1.59 g$ & $659 \pm 2.14 \mathrm{~cd}$ \\
\hline Şire & $453 \pm 0.92 c$ & $146 \pm 0.61 c$ & $598 \pm 5.49 d$ & $556 \pm 6.58 a$ & $130 \pm 6.27 c$ & $583 \pm 2.83 e$ \\
\hline Tahannebi & $465 \pm 1.68 b$ & $186 \pm 2.87 a$ & $582 \pm 9.36 \mathrm{ef}$ & $444 \pm 2.36 c$ & $170 \pm 1.54 a$ & $660 \pm 4.95 \mathrm{~cd}$ \\
\hline Zeyni & $296 \pm 2.42 \mathrm{i}$ & $34 \pm 2.58 \mathrm{k}$ & $559 \pm 2.50 \mathrm{~g}$ & $344 \pm 1.01 \mathrm{~g}$ & $88 \pm 1.26 \mathrm{~g}$ & $580 \pm 1.76 e$ \\
\hline Mean & 297.00 & 92.00 & 555.00 & 349.00 & 100.00 & 599.00 \\
\hline $\mathrm{F}_{0.05}$ & 4040.72 & 639.69 & 862.62 & 1284.49 & 128.47 & 4800.83 \\
\hline \multicolumn{7}{|c|}{ Main Effects } \\
\hline Varieties & \multicolumn{2}{|c|}{ Skin } & \multicolumn{2}{|c|}{ Flesh } & \multicolumn{2}{|c|}{ Seed } \\
\hline Ağ Banki & \multicolumn{2}{|c|}{$421 \pm 0.70 \mathrm{e}$} & \multicolumn{2}{|c|}{$51 \pm 0.86 \mathrm{~g}$} & \multicolumn{2}{|c|}{$364 \pm 1.40 \mathrm{~h}$} \\
\hline Azezi & \multicolumn{2}{|c|}{$220 \pm 0.611$} & \multicolumn{2}{|c|}{$145 \pm 1.06 b$} & \multicolumn{2}{|c|}{$607 \pm 3.33 c$} \\
\hline Chardonnay & \multicolumn{2}{|c|}{$429 \pm 0.81 d$} & \multicolumn{2}{|c|}{$56 \pm 0.40 \mathrm{~g}$} & \multicolumn{2}{|c|}{$632 \pm 0.98 b$} \\
\hline Çiloreş & & & $54 \pm 0$ & & $731 \pm$ & $20 a$ \\
\hline Çilorut & & & $71 \pm 0$ & & $548 \pm 4$ & $84 \mathrm{ef}$ \\
\hline Elma Üzümü & & & $72 \pm 0$ & & $610 \pm$ & $90 c$ \\
\hline Hasani & & & $55 \pm 0$ & & $539 \pm$ & $.93 f$ \\
\hline Hatun Parmağı & & & $74 \pm 0$ & & $559 \pm$ & $68 \mathrm{e}$ \\
\hline Kabarcık & & & $98 \pm 0.2$ & & $542 \pm$ & $.50 f$ \\
\hline Küllahi & 201 & & $110 \pm 0$ & & $522 \pm 2$ & $91 \mathrm{~g}$ \\
\hline Perlette & & & $135 \pm 2$ & & Seed & \\
\hline Serpenekıran & & & $122 \pm 2$ & & $610 \pm$ & $.06 c$ \\
\hline Simore & & & $105 \pm 0$ & & $581 \pm$ & $02 d$ \\
\hline Su Üzümü & & & $106 \pm 0$ & & $626 \pm 4$ & $45 \mathrm{bc}$ \\
\hline Şeffafi & & & $96 \pm 0.1$ & & $557 \pm$ & $95 \mathrm{e}$ \\
\hline Şire & & & $138 \pm 3$ & & $590 \pm 2$ & $80 \mathrm{~cd}$ \\
\hline Tahannebi & & & $178 \pm 1$ & & $621 \pm 7$ & $14 \mathrm{bc}$ \\
\hline Zeyni & 32 & & $61 \pm 0.7$ & & $570 \pm 1$ & 92de \\
\hline Mean & & & 96.0 & & 577 & \\
\hline $\mathrm{F}_{0.05}$ & & & 424. & & 246 & \\
\hline
\end{tabular}

Values marked with different letters in the same column were statistically significant at $p<0.05$ level according to the Duncan's test. Main effects were grouped as mean of two years, and interactions were grouped within the year to which they belong. All results expressed as mean of repetitions \pm standard error. 
Table 3. Total phenolic compound contents of colored grapes

\begin{tabular}{|c|c|c|c|c|c|c|}
\hline \multirow{3}{*}{ Varieties } & \multicolumn{6}{|c|}{ Total Phenolic Compound $\left(\mathrm{mg} \mathrm{kg}^{-1}\right)$} \\
\hline & \multicolumn{3}{|c|}{2013} & \multicolumn{3}{|c|}{2014} \\
\hline & Skin & Flesh & Seed & Skin & Flesh & Seed \\
\hline Cabernet Sauvignon & $375 \pm 3.55 f$ & $154 \pm 2.72 c$ & $594 \pm 6.07 c$ & $466 \pm 2.61 \mathrm{e}$ & $127 \pm 1.46 d$ & $569 \pm 1.87 e$ \\
\hline Horoz Karası & $465 \pm 1.45 b$ & $174 \pm 1.20 \mathrm{a}$ & $717 \pm 3.19 b$ & $544 \pm 1.32 b$ & $170 \pm 0.30 a$ & $817 \pm 4.84 b$ \\
\hline Hönüsü & $423 \pm 2.56 d$ & $78 \pm 0.85 i$ & $492 \pm 1.50 \mathrm{~g}$ & $448 \pm 2.17 \mathrm{~g}$ & $122 \pm 1.52 d$ & $535 \pm 2.04 f$ \\
\hline Kara Kabarcık & $476 \pm 3.42 a$ & $123 \pm 3.11 f$ & $561 \pm 2.90 \mathrm{e}$ & $545 \pm 1.76 b$ & $112 \pm 1.60 \mathrm{e}$ & $585 \pm 2.92 d$ \\
\hline Kızıl Banki & $362 \pm 1.01 \mathrm{~g}$ & $137 \pm 3.10 \mathrm{e}$ & $537 \pm 5.04 f$ & $371 \pm 2.05 i$ & $140 \pm 1.37 c$ & $583 \pm 0.83 d$ \\
\hline Öküzgözü & $426 \pm 1.26 d$ & $167 \pm 1.89 b$ & $462 \pm 3.39 h$ & $564 \pm 0.80 a$ & $153 \pm 1.54 b$ & $582 \pm 1.70 d$ \\
\hline Sergi Karası & $403 \pm 3.61 \mathrm{e}$ & $152 \pm 0.80 d$ & $574 \pm 6.43 \mathrm{de}$ & $545 \pm 2.88 b$ & $144 \pm 3.73 b c$ & $574 \pm 2.66 \mathrm{de}$ \\
\hline Syrah & $464 \pm 2.89 b$ & $76 \pm 1.75 i$ & $1247 \pm 6.70 \mathrm{a}$ & $534 \pm 1.89 c$ & $101 \pm 2.26 f$ & $1119 \pm 1.84 a$ \\
\hline Tilgören & $435 \pm 2.81 c$ & $95 \pm 2.47 \mathrm{~h}$ & $574 \pm 3.24$ de & $494 \pm 3.84 d$ & $105 \pm 0.83 \mathrm{ef}$ & $575 \pm 2.67 \mathrm{de}$ \\
\hline Top Üzüm & $364 \pm 1.52 \mathrm{~g}$ & $103 \pm 2.02 \mathrm{~g}$ & $578 \pm 9.72 d$ & $417 \pm 1.58 \mathrm{~h}$ & $123 \pm 1.52 d$ & $608 \pm 0.91 c$ \\
\hline Yediveren & $367 \pm 0.82 \mathrm{fg}$ & $59 \pm 0.82 j$ & $544 \pm 1.13 f$ & $457 \pm 4.72 f$ & $63 \pm 2.25 \mathrm{~g}$ & $586 \pm 2.31 d$ \\
\hline Mean & 415.00 & 120.00 & 625.00 & 490.00 & 124.00 & 648.00 \\
\hline $\mathrm{F}_{0.05}$ & 290.19 & 357.29 & 1632.16 & 573.94 & 160.22 & 6729.00 \\
\hline \multicolumn{7}{|c|}{ Main Effects } \\
\hline Varieties & \multicolumn{2}{|r|}{ Skin } & \multicolumn{2}{|c|}{ Flesh } & \multicolumn{2}{|c|}{ Seed } \\
\hline Cabernet Sauvignon & \multicolumn{2}{|c|}{$421 \pm 1.64 \mathrm{~g}$} & \multicolumn{2}{|c|}{$140 \pm 0.63 c$} & \multicolumn{2}{|c|}{$582 \pm 3.39 c$} \\
\hline Horoz Karası & \multicolumn{2}{|c|}{$505 \pm 0.88 b$} & \multicolumn{2}{|c|}{$172 \pm 0.74 a$} & \multicolumn{2}{|c|}{$767 \pm 3.02 b$} \\
\hline Hönüsü & \multicolumn{2}{|c|}{$436 \pm 1.59 f$} & \multicolumn{2}{|c|}{$100 \pm 0.90 \mathrm{e}$} & \multicolumn{2}{|c|}{$513 \pm 0.30 \mathrm{~g}$} \\
\hline Kara Kabarcık & \multicolumn{2}{|c|}{$511 \pm 1.30 a$} & \multicolumn{2}{|c|}{$118 \pm 1.78 d$} & \multicolumn{2}{|c|}{$573 \pm 0.03 d$} \\
\hline Kızıl Banki & \multicolumn{2}{|c|}{$366 \pm 0.63 j$} & \multicolumn{2}{|c|}{$139 \pm 2.22 c$} & \multicolumn{2}{|c|}{$560 \pm 2.23 e$} \\
\hline Öküzgözü & \multicolumn{2}{|c|}{$495 \pm 1.03 c$} & \multicolumn{2}{|c|}{$160 \pm 0.29 b$} & \multicolumn{2}{|c|}{$522 \pm 1.51 f$} \\
\hline Sergi Karası & \multicolumn{2}{|c|}{$474 \pm 3.07 d$} & \multicolumn{2}{|c|}{$148 \pm 1.90 c$} & \multicolumn{2}{|c|}{$574 \pm 3.55 d$} \\
\hline Syrah & \multicolumn{2}{|c|}{$499 \pm 0.87 c$} & & $80 f$ & & $33 \pm 2.62 a$ \\
\hline Tilgören & & $4 \pm 1.76 \mathrm{e}$ & & $49 \mathrm{e}$ & & $5 \pm 2.44 d$ \\
\hline Top Üzüm & & $0 \pm 0.11 \mathrm{i}$ & & 39de & & $3 \pm 4.63 c$ \\
\hline Yediveren & & $2 \pm 2.06 \mathrm{~h}$ & & $83 g$ & & $5 \pm 1.19 e$ \\
\hline Mean & & 452.00 & & & & 637.00 \\
\hline $\mathrm{F}_{0.05}$ & & 950.40 & & & & 870.76 \\
\hline
\end{tabular}

Values marked with different letters in the same column were statistically significant at $p<0.05$ level according to the Duncan's test. Main effects were grouped as mean of two years, and interactions were grouped within the year to which they belong. All results expressed as mean of repetitions \pm standard error.

year and variety (Yayla, 2008). The amount of phenolic compounds in colored varieties is higher than in white varieties. The reason for this is that anthocyanins increase the total amount of phenolic compounds (Kaur and Kapoor 2001). Dávalos et al. (2005) have reported that the total amount of phenolic compounds was higher in red grape juice than in other samples based on the results of their study conducted in red and white grape juices and vinegar.

Grape varieties differ in terms of the amount of phenolic compounds. These differences are due to the differences such as water content, size, skin thickness, number of seeds and size of the seed. In this study, the differences observed between years are determined to be due to the differences in climate difference between the years.

\section{Total Anthocyanin Contents}

When we examined the average of both years of white grape varieties, we determined that the total anthocyanin content of the skin was $0.322 \mathrm{mg} \mathrm{kg}^{-1}$ in the Azezi being the lowest and $1.981 \mathrm{mg} \mathrm{kg}^{-1}$ in the Tahannebi being the highest. We determined that the total anthocyanin content of the fruit flesh was $0.099 \mathrm{~g} \mathrm{~kg} \mathrm{k}^{-1}$ in the Kabarcık as the lowest value, and $0.528 \mathrm{mg} \mathrm{kg}^{-1}$ in the Chardonnay as the highest value. Besides, Çilorut was determined to have the lowest total anthocyanin content in the seed with the value of $2.48 \mathrm{mg} \mathrm{kg}^{-1}$ and Serpenekıran was found to have the highest content with the value of $7.23 \mathrm{mg} \mathrm{kg}^{-1}$ (Table 4). While the average yearly average of the colored grape varieties was the Kızıl Banki with the lowest $59 \mathrm{mg} \mathrm{kg}^{-1}$ in terms of total anthocyanin 
Table 4. Total anthocyanin contents of white grapes

\begin{tabular}{|c|c|c|c|c|c|c|}
\hline \multirow{3}{*}{ Varieties } & \multicolumn{6}{|c|}{ Total Anthocyanin $\left(\mathrm{mg} \mathrm{kg}^{-1}\right)$} \\
\hline & \multicolumn{3}{|c|}{2013} & \multicolumn{3}{|c|}{2014} \\
\hline & Skin & Flesh & Seed & Skin & Flesh & Seed \\
\hline Ağ Banki & $0.728 \pm 0.01 \mathrm{e}$ & $0.252 \pm 0.01 \mathrm{gh}$ & $4.62 \pm 0.26 \mathrm{de}$ & $0.721 \pm 0.02 \mathrm{fgh}$ & $0.246 \pm 0.03 c$ & $4.24 \pm 0.12 d$ \\
\hline Azezi & $0.266 \pm 0.02 k$ & $0.356 \pm 2.00 \mathrm{def}$ & $3.59 \pm 0.06 f$ & $0.378 \pm 0.09 j$ & $0.328 \pm 0.01 c$ & $3.76 \pm 0.19 \mathrm{de}$ \\
\hline Chardonnay & $0.560 \pm 0.03 \mathrm{ghi}$ & $0.507 \pm 0.59 a$ & $4.53 \pm 0.10 \mathrm{e}$ & $0.513 \pm 0.19 \mathrm{hi}$ & $0.550 \pm 0.01 a$ & $4.39 \pm 0.09 \mathrm{~cd}$ \\
\hline Çiloreş & $0.383 \pm 0.05 j$ & $0.179 \pm 1.32 \mathrm{ij}$ & $4.52 \pm 0.10 \mathrm{e}$ & $0.447 \pm 0.02 i$ & $0.196 \pm 0.01 d$ & $4.34 \pm 0.19 \mathrm{~cd}$ \\
\hline Çilorut & $0.676 \pm 0.03 f$ & $0.278 \pm 1.10 \mathrm{fg}$ & $2.63 \pm 0.03 \mathrm{~h}$ & $0.731 \pm 0.06 \mathrm{fgh}$ & $0.266 \pm 0.01 \mathrm{c}$ & $2.33 \pm 0.14 f$ \\
\hline Elma Üzümü & $1.036 \pm 0.03 b$ & $0.479 \pm 1.24 b$ & $4.94 \pm 0.06 d$ & $0.857 \pm 0.02 \mathrm{ef}$ & $0.482 \pm 0.0 \mathrm{~b}$ & $4.77 \pm 0.05 c$ \\
\hline Hasani & $0.983 \pm 0.09 c$ & $0.302 \pm 1.62 f$ & $3.35 \pm 0.07 f$ & $0.854 \pm 0.13$ ef & $0.293 \pm 0.01 \mathrm{c}$ & $3.20 \pm 0.07 e$ \\
\hline Hatun Parmağı & $0.453 \pm 0.03 i$ & $0.160 \pm 1.35 \mathrm{j}$ & $6.12 \pm 0.11 b$ & $0.545 \pm 0.08 \mathrm{~h}$ & $0.140 \pm 0.01 d$ & $5.51 \pm 0.06 b$ \\
\hline Kabarcık & $1.950 \pm 0 a$ & $0.102 \pm 1.201$ & $3.43 \pm 0.10 f$ & $1.941 \pm 0.13 a b$ & $0.097 \pm 0.28 \mathrm{e}$ & $3.90 \pm 0.17 \mathrm{de}$ \\
\hline Küllahi & $0.495 \pm 0.28 \mathrm{i}$ & $0.118 \pm 1.98 \mathrm{k}$ & $3.08 \pm 0.10 \mathrm{~g}$ & $0.533 \pm 0.06 h$ & $0.130 \pm 0.01 \mathrm{de}$ & $3.28 \pm 0.32 \mathrm{e}$ \\
\hline Perlette & $0.982 \pm 0.09 c$ & $0.301 \pm 2.51 f$ & Seedless & $0.846 \pm 0.09$ ef & $0.294 \pm 0.02 c$ & Seedless \\
\hline Serpenekıran & $1.015 \pm 0.18 b c$ & $0.442 \pm 3.40 \mathrm{~cd}$ & $7.17 \pm 0.07 a$ & $1.062 \pm 0 \mathrm{bc}$ & $0.453 \pm 0.01 b$ & $7.29 \pm 0.16 a$ \\
\hline Simore & $0.943 \pm 0.19 d$ & $0.458 \pm 1.30 \mathrm{bc}$ & $3.25 \pm 0.13 f$ & $0.815 \pm 0.04 \mathrm{efg}$ & $0.471 \pm 0.01 b$ & $3.04 \pm 0.14 \mathrm{e}$ \\
\hline Su Üzümü & $1.030 \pm 0.12 b$ & $0.437 \pm 1.49$ cde & $7.15 \pm 0.10 a$ & $1.045 \pm 0.03 \mathrm{~cd}$ & $0.427 \pm 0.02 b c$ & $6.84 \pm 0.17 a b$ \\
\hline Şeffafi & $0.608 \pm 0.08 \mathrm{~g}$ & $0.241 \pm 1.49 \mathrm{gh}$ & $4.93 \pm 0.06 d$ & $0.632 \pm 0.33 \mathrm{gh}$ & $0.252 \pm 0.0 c$ & $4.88 \pm 0.12 c$ \\
\hline Şire & $0.964 \pm 0.19 \mathrm{~cd}$ & $0.500 \pm 0.61 a$ & $7.05 \pm 0.07 a$ & $0.970 \pm 0.03 \mathrm{de}$ & $0.517 \pm 0.02 \mathrm{ab}$ & $7.17 \pm 0.33 a$ \\
\hline Tahannebi & $1.943 \pm 0.13 a$ & $0.457 \pm 2.87 \mathrm{bc}$ & $5.44 \pm 0.19 c$ & $2.020 \pm 0.21 a$ & $0.448 \pm 0.01 b$ & $5.46 \pm 0.24 b$ \\
\hline Zeyni & $0.520 \pm 0.05 \mathrm{hi}$ & $0.189 \pm 2.58 \mathrm{i}$ & $4.53 \pm 0.10 \mathrm{e}$ & $0.499 \pm 0.02 i$ & $0.194 \pm 0.03 d$ & $4.62 \pm 0.05 c$ \\
\hline Mean & 0.86 & 0.32 & 4.73 & 0.85 & 0.32 & 4.65 \\
\hline $\mathrm{F}_{0.05}$ & 35.05 & 8.96 & 271.61 & 35.37 & 3.91 & 199.58 \\
\hline \multicolumn{7}{|c|}{ Main Effects } \\
\hline Varieties & \multicolumn{2}{|r|}{ Skin } & \multicolumn{2}{|c|}{ Flesh } & \multicolumn{2}{|c|}{ Seed } \\
\hline Ağ Banki & \multicolumn{2}{|c|}{$0.725 \pm 0.01 \mathrm{fg}$} & \multicolumn{2}{|c|}{$0.249 \pm 0.02 \mathrm{ef}$} & \multicolumn{2}{|c|}{$4.43 \pm 0.07 b c$} \\
\hline Azezi & \multicolumn{2}{|c|}{$0.322 \pm 0.05 k$} & \multicolumn{2}{|c|}{$0.342 \pm 0.02 d$} & \multicolumn{2}{|c|}{$3.67 \pm 0.12 \mathrm{bcd}$} \\
\hline Chardonnay & \multicolumn{2}{|c|}{$0.537 \pm 0.09 h$} & \multicolumn{2}{|c|}{$0.528 \pm 0.01 a$} & \multicolumn{2}{|c|}{$4.46 \pm 0.06 b c$} \\
\hline Çiloreş & \multicolumn{2}{|c|}{$0.415 \pm 0.04 j$} & \multicolumn{2}{|c|}{$0.188 \pm 0.01 \mathrm{fg}$} & \multicolumn{2}{|c|}{$4.43 \pm 0.14 b c$} \\
\hline Çilorut & \multicolumn{2}{|c|}{$0.703 \pm 0.02 \mathrm{fg}$} & \multicolumn{2}{|c|}{$0.272 \pm 0.06 \mathrm{e}$} & \multicolumn{2}{|c|}{$2.48 \pm 0.06 \mathrm{e}$} \\
\hline Elma Üzümü & & $46 \pm 0.01 \mathrm{de}$ & & $\pm 0.02 \mathrm{~b}$ & 4.86 & $0.04 b$ \\
\hline Hasani & & $18 \pm 0.07$ ef & & $3 \pm 0.01 \mathrm{e}$ & $3.28 \pm$ & $04 \mathrm{cde}$ \\
\hline Hatun Parmağı & & $199 \pm 0.05 i$ & & $\pm 0.02 \mathrm{fg}$ & $5.81 \pm$ & $.09 a b$ \\
\hline Kabarcık & & $45 \pm 0.06 b$ & & $9 \pm 0.13 \mathrm{~h}$ & $3.66 \pm$ & $04 \mathrm{bcd}$ \\
\hline Küllahi & & $14 \pm 0.13 \mathrm{hi}$ & & $\pm 0.0 \mathrm{gh}$ & $3.18 \pm$ & $20 \mathrm{cde}$ \\
\hline Perlette & & $14 \pm 0.04 \mathrm{ef}$ & & $7 \pm 0.02 \mathrm{e}$ & See & less \\
\hline Serpenekıran & & $38 \pm 0.09 c$ & & $8 \pm 0.0 c$ & 7.23 & $0.11 \mathrm{a}$ \\
\hline Simore & 0.8 & $9 \pm 0.10 \mathrm{efg}$ & & $\pm 0.02 \mathrm{bc}$ & $3.15 \pm$ & $11 \mathrm{cde}$ \\
\hline Su Üzümü & & $38 \pm 0.06 c$ & & $2 \pm 0.03 c$ & 6.99 & $0.13 a$ \\
\hline Şeffafi & & $20 \pm 0.15 \mathrm{~g}$ & & $\pm 0.02 \mathrm{ef}$ & 4.91 & $0.08 b$ \\
\hline Şire & 0.9 & $7 \pm 0.11 \mathrm{cde}$ & & $\pm 0.03 \mathrm{ab}$ & 7.11 & $0.18 a$ \\
\hline Tahannebi & & $81 \pm 0.11 a$ & & $\pm 0.04 \mathrm{bc}$ & $5.45 \pm$ & $.07 a b$ \\
\hline Zeyni & & $10 \pm 0.03 \mathrm{hi}$ & & $\pm 0.01 \mathrm{fg}$ & $4.58 \pm$ & $.03 b c$ \\
\hline Mean & & 0.86 & & .32 & & \\
\hline $\mathrm{F}_{0.05}$ & & 47.99 & & .56 & & \\
\hline
\end{tabular}

Values marked with different letters in the same column were statistically significant at $p<0.05$ level according to the Duncan's test. Main effects were grouped as mean of two years, and interactions were grouped within the year to which they belong. All results expressed as mean of repetitions \pm standard error. 
content in skin, it was observed that the Kara Kabarcık had the highest value with $1370 \mathrm{mg} \mathrm{kg}^{-}$ 1 . On the other hand, the total anthocyanin content of the fruit flesh was found to be 0.354 $\mathrm{mg} \mathrm{kg}^{-1}$ in the Kızıl Banki as the lowest, and the highest was $5.67 \mathrm{mg} \mathrm{kg}^{-1}$ in the Top Üzüm. The total anthocyanin content of the seeds in colored grape varieties ranged from 3.08 to $9.05 \mathrm{mg} \mathrm{kg}^{-}$ 1 , and the lowest value was determined in Sergi Karası, whilst the highest value was determined in Öküzgözü (Table 5).

The most important group of phenolic compounds found in grapes are anthocyanins which are responsible for color (Cemeroglu et al., 2001). Anthocyanins are natural color pigments that give their distinctive red, blue and purple hues (Costa et al., 2000; Camire et al., 2002). Anthocyanins are a very large and important subgroup of phenolic substances and they start to form in the veraison phase in grapes, accumulate during ripening phase and reach the highest level after maturity (Kunter et al., 2013).

Cangi et al. (2011) reported that the amount of anthocyanin in grapes increased in parallel with the color change. Also, Fernandez Lopez et al. (1992) demonstrated that the anthocyanin content in grapes was $310 \mathrm{mg} \mathrm{kg}^{-1}$ at the beginning of maturity and it was $1140 \mathrm{mg}$ $\mathrm{kg}^{-1}$ during the maturity period. Similarly, Revilla et al. (2001) showed in their study that the total amount of anthocyanin varied between 273-804 $\mathrm{mg} \mathrm{kg}^{-1}$ in Cabernet Sauvignon grapes and 218$693 \mathrm{mg} \mathrm{kg}^{-1}$ in Tempranillo grapes during maturity and increased with maturity.

Previous studies have shown that the ecological conditions have also great impact on the anthocyanin content of grapes. Toprak, (2011) compared the anthocyanin content of the Kalecik Karasi cultured in four different ecology (Ankara - Kalecik, Ankara - Keçiören, Ankara -

Table 5. Total anthocyanin contents of colored grapes

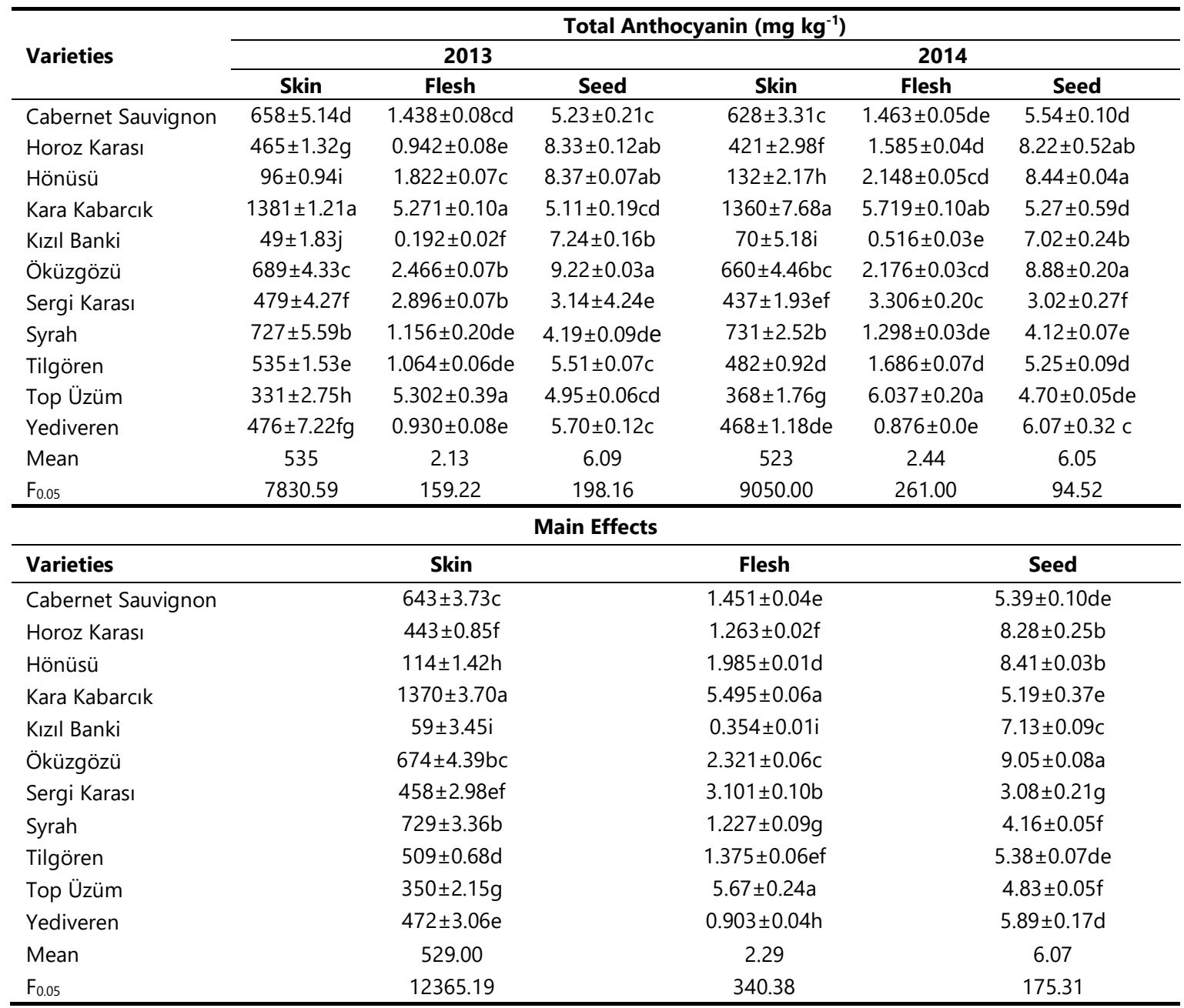

Values marked with different letters in the same column were statistically significant at $p<0.05$ level according to the Duncan's test. Main effects were grouped as mean of two years, and interactions were grouped within the year to which they belong. All results expressed as mean of repetitions \pm standard error. 
Polatlı and Nevşehir - Çat) and he reported that for Kalecik the total anthocyanin content was $202 \mathrm{mg} \mathrm{kg}^{-1}$, for Keçiören $196 \mathrm{mg} \mathrm{kg}^{-1}$, for Polatı $319 \mathrm{mg} \mathrm{kg}^{-1}$ and for Çat $299 \mathrm{mg} \mathrm{kg}^{-1}$.

\section{Total Tannin Contents}

The average total tannin content of the skin of the white grape varieties varies between $197 \mathrm{mg} \mathrm{kg}^{-1}$ and $740 \mathrm{mg} \mathrm{kg}^{-1}$, while the total

Table 6. Total tannin contents of white grapes

\begin{tabular}{|c|c|c|c|c|c|c|}
\hline \multirow{3}{*}{ Varieties } & \multicolumn{6}{|c|}{ Total Tannin (mg kg-1) } \\
\hline & \multicolumn{3}{|c|}{2013} & \multicolumn{3}{|c|}{2014} \\
\hline & Skin & Flesh & Seed & Skin & Flesh & Seed \\
\hline Ağ Banki & $427 \pm 7.71 \mathrm{~g}$ & $131 \pm 2.53 b$ & $1458 \pm 5.93 j$ & $384 \pm 1.78 e$ & $120 \pm 2.31 c$ & $1309 \pm 27.45 g$ \\
\hline Azezi & $237 \pm 5.79 i j$ & $114 \pm 1.27 d$ & $1612 \pm 6.07$ ef & $211 \pm 7.88 \mathrm{~g}$ & $100 \pm 1.96 f$ & $1569 \pm 7.49 e$ \\
\hline Chardonnay & $501 \pm 2.59 \mathrm{de}$ & $109 \pm 1.12 \mathrm{de}$ & $1558 \pm 1.40 \mathrm{hi}$ & $484 \pm 17.73 d$ & $122 \pm 0.72 b c$ & $1561 \pm 6.15 e$ \\
\hline Çiloreş & $750 \pm 3.08 a$ & $100 \pm 4.85 e$ & $1630 \pm 9.33 \mathrm{def}$ & $729 \pm 32.42 a$ & $104 \pm 2.09 e$ & $1592 \pm 7.41 \mathrm{~cd}$ \\
\hline Çilorut & $579 \pm 12.92 c$ & $40 \pm 1.64 j$ & $1614 \pm 15.99 d e f$ & $593 \pm 16.99 b$ & $50 \pm 0.49 j$ & $1580 \pm 9.40 d$ \\
\hline Elma Üzümü & $317 \pm 2.67 \mathrm{~h}$ & $100 \pm 1.59 f$ & $1599 \pm 3.33 f g h$ & $260 \pm 1.49 f$ & $94 \pm 1.20 \mathrm{gh}$ & $1623 \pm 2.50 b c$ \\
\hline Hasani & $486 \pm 4.92 e$ & $77 \pm 0.48 \mathrm{~h}$ & $1636 \pm 6.19 \mathrm{def}$ & $460 \pm 12.99 d$ & $75 \pm 1.65 i$ & $1590 \pm 2.23 \mathrm{~cd}$ \\
\hline Hatun Parmağı & $581 \pm 2.21 c$ & $60 \pm 1.90 \mathrm{i}$ & $1888 \pm 42.83 a$ & $532 \pm 14.00 c$ & $48 \pm 1.20 \mathrm{j}$ & $1888 \pm 30.63 a$ \\
\hline Kabarcık & $245 \pm 3.60 \mathrm{i}$ & $161 \pm 2.04 a$ & $1658 \pm 4.13 \mathrm{~cd}$ & $261 \pm 14.07 f$ & $182 \pm 3.32 a$ & $1602 \pm 1.92 c$ \\
\hline Küllahi & $502 \pm 4.63 \mathrm{de}$ & $59 \pm 2.35 i$ & $1594 \pm 6.91 \mathrm{fgh}$ & $478 \pm 13.46 d$ & $46 \pm 1.15 j$ & $1574 \pm 15.17 \mathrm{de}$ \\
\hline Perlette & $605 \pm 4.08 b$ & $91 \pm 2.56 f g$ & Seedless & $547 \pm 1.48 b c$ & $109 \pm 1.31 \mathrm{de}$ & Seedless \\
\hline Serpenekıran & $319 \pm 4.36 h$ & $92 \pm 1.25 \mathrm{fg}$ & $1645 \pm 4.54 \mathrm{de}$ & $280 \pm 17.88 f$ & $97 \pm 0.40 f g$ & $1604 \pm 7.25 c$ \\
\hline Simore & $617 \pm 4.45 b$ & $110 \pm 3.29 e$ & $1576 \pm 10.53 \mathrm{gh}$ & $560 \pm 17.40 b c$ & $90 \pm 1.33 \mathrm{~h}$ & $1587 \pm 10.11 \mathrm{~cd}$ \\
\hline Su Üzümü & $518 \pm 10.71 d$ & $116 \pm 2.55 d$ & $1594 \pm 6.51 \mathrm{fgh}$ & $468 \pm 13.11 d$ & $126 \pm 0.91 b$ & $1577 \pm 12.0 d$ \\
\hline Şeffafi & $509 \pm 8.40 d$ & $81 \pm 3.18 \mathrm{~h}$ & $1591 \pm 11.74 f g$ & $439 \pm 8.44 d$ & $92 \pm 1.91 \mathrm{~h}$ & $1561 \pm 3.05 e$ \\
\hline Şire & $221 \pm 2.47 \mathrm{j}$ & $130 \pm 2.91 c$ & $1700 \pm 15.00 b$ & $174 \pm 10.76 g$ & $110 \pm 1.71 \mathrm{de}$ & $1637 \pm 38.39 b$ \\
\hline Tahannebi & $509 \pm 1.58 d$ & $107 \pm 0.69 e$ & $1683 \pm 14.47 \mathrm{bc}$ & $459 \pm 28.47 d$ & $111 \pm 0.73 d$ & $1586 \pm 2.43 \mathrm{~cd}$ \\
\hline Zeyni & $461 \pm 1.91 f$ & $90 \pm 1.04 \mathrm{~g}$ & $1529 \pm 5.61 i$ & $437 \pm 6.23 d$ & $91 \pm 1.66 h$ & $1448 \pm 18.74 f$ \\
\hline Mean & 466.00 & 98.00 & 1621.00 & 431.00 & 98.00 & 1581.00 \\
\hline $\mathrm{F}_{0.05}$ & 634.76 & 156.39 & 925.62 & 86.33 & 404.29 & 611.24 \\
\hline \multicolumn{7}{|c|}{ Main Effects } \\
\hline Varieties & \multicolumn{2}{|c|}{ Skin } & \multicolumn{2}{|c|}{ Flesh } & \multicolumn{2}{|r|}{ Seed } \\
\hline Ağ Banki & \multicolumn{2}{|c|}{$405 \pm 3.06 f$} & \multicolumn{2}{|c|}{$126 \pm 2.36 b$} & \multicolumn{2}{|r|}{$1384 \pm 10.86 k$} \\
\hline Azezi & \multicolumn{2}{|c|}{$224 \pm 2.37 i$} & \multicolumn{2}{|c|}{$107 \pm 1.61 \mathrm{e}$} & \multicolumn{2}{|r|}{$1591 \pm 4.92 \mathrm{fgh}$} \\
\hline Chardonnay & \multicolumn{2}{|c|}{$493 \pm 8.47 d$} & \multicolumn{2}{|c|}{$116 \pm 0.82 d$} & \multicolumn{2}{|r|}{$1560 \pm 2.95 i$} \\
\hline Çiloreş & \multicolumn{2}{|c|}{$740 \pm 15.75 a$} & \multicolumn{2}{|c|}{$102 \pm 2.11 \mathrm{e}$} & \multicolumn{2}{|r|}{$1611 \pm 8.20$ defg } \\
\hline Çilorut & \multicolumn{2}{|c|}{$586 \pm 7.76 b$} & \multicolumn{2}{|c|}{$45 \pm 0.59 k$} & \multicolumn{2}{|r|}{$1597 \pm 4.31$ efg } \\
\hline Elma Üzümü & 289 & $1.38 \mathrm{~g}$ & & $39 g$ & & $1611 \pm 2.50 \mathrm{defg}$ \\
\hline Hasani & 473 & $4.23 d$ & & $.04 i$ & & $1613 \pm 4.15 \mathrm{def}$ \\
\hline Hatun Parmağı & 556 & $6.53 c$ & & $.79 \mathrm{j}$ & & $1888 \pm 18.94 a$ \\
\hline Kabarcık & 253 & $5.61 \mathrm{~h}$ & & $2.02 a$ & & $1630 \pm 2.86 c d$ \\
\hline Küllahi & 490 & $3.19 d$ & & $.47 \mathrm{j}$ & & $1584 \pm 10.99 f g h$ \\
\hline Perlette & $576 \pm$ & $.91 b c$ & & $1.87 f$ & & Seedless \\
\hline Serpenekıran & $299 \pm$ & $0.31 \mathrm{~g}$ & & $59 g$ & & $1625 \pm 4.30$ cde \\
\hline Simore & 589 & $9.82 b$ & & $.12 \mathrm{fg}$ & & $1582 \pm 8.09 f g h i$ \\
\hline Su Üzümü & 493 & $3.73 d$ & & $1.69 c$ & & $1586 \pm 9.25 f g h i$ \\
\hline Şeffafi & 474 & $7.58 d$ & & $.37 \mathrm{~h}$ & & $1576 \pm 4.81 \mathrm{ghi}$ \\
\hline Şire & 197 & 4.33j & & $.05 c d$ & & $1669 \pm 17.22 b$ \\
\hline Tahannebi & $484 \pm$ & $3.75 d$ & & $.27 e$ & & $1635 \pm 8.40 c$ \\
\hline Zeyni & 449 & $4.06 \mathrm{e}$ & & $34 \mathrm{~h}$ & & $1489 \pm 8.06 j$ \\
\hline Mean & & & & & & 1602.00 \\
\hline $\mathrm{F}_{0.05}$ & & & & 28 & & 2215.40 \\
\hline
\end{tabular}

Values marked with different letters in the same column were statistically significant at $p<0.05$ level according to the Duncan's test. Main effects were grouped as mean of two years, and interactions were grouped within the year to which they belong. All results expressed as mean of repetitions \pm standard error. 
tannin content of the fruit flesh is between 45 $\mathrm{mg} \mathrm{kg}^{-1}$ and $172 \mathrm{mg} \mathrm{kg}^{-1}$. On the other hand, the total tannin content of the seed was observed to range between $1384 \mathrm{mg} \mathrm{kg}^{-1}$ and $1888 \mathrm{mg} \mathrm{kg}^{-1}$ (Table 6).

As a result of the statistical analysis performed between the varieties by calculating the average of both years of the colored varieties, we determined that the difference between the varieties is significant. Kızıl Banki had the lowest value of the total tannin content in the skin, while this value in Kara Kabarcık was the highest. Besides, it was determined that the total tannin content of the fruit flesh ranged between 88 and $234 \mathrm{mg} \mathrm{kg}^{-1}$, a"nd the total tannin content of the seed ranged between 1478 and $1659 \mathrm{mg} \mathrm{kg}^{-1}$ (Table 7).

In addition to the differences in tannin content of different varieties, it was observed that different climatic conditions in different years had also effects on the tannin content of the grapes. In addition, significant differences were observed in terms of the ratio of tannins contained in the skin, fruit flesh and seeds of the grape varieties.

The tannins found in grape skins have a larger structure than the ones in the seed. As the degree of polymerization of tannins increases, their reactivity potential with proteins also enhances. The polymeric tannins in the skin of the grape react with the proteins and make the cell wall tighter and thus the grape gains resistance against external factors. Tannins are mostly located in the inner cells of the skin (Gagne et al., 2006). Souquet et al. (2000) reported that the amount of tannin in the pedicel is between the amount of tannin in the skin and the amount of tannins in the seed, and that the

Table 7. Total tannin contents of colored grapes

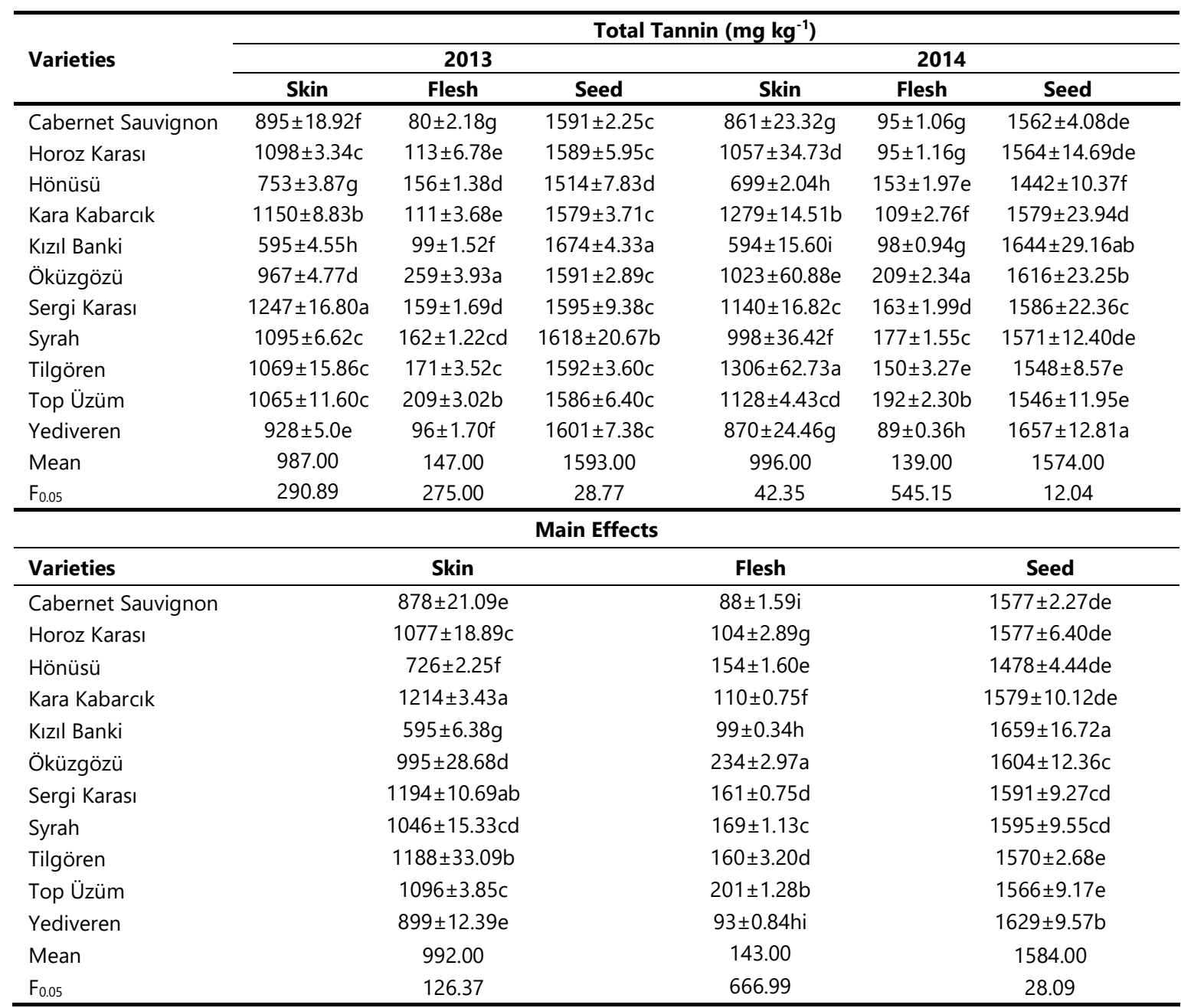

Values marked with different letters in the same column were statistically significant at $p<0.05$ level according to the Duncan's test. Main effects were grouped as mean of two years, and interactions were grouped within the year to which they belong. All results expressed as mean of repetitions \pm standard error. 
grape being red or white does not affect the amount of tannins in the pedicel. Harbertson et al. (2002)singleton stated that the amount of tannin in the seed is approximately three times higher than in the skin. Torchio et al. (2010) found that the amount of tannin in the skin of Barbera grapes with different sugar levels grown in various vineyards was $595-1196 \mathrm{mg} \mathrm{kg}^{-1}$, and the amount of tannins in the seed was between $516-1092 \mathrm{mg} \mathrm{kg}^{-1}$. Peng et al. (2001) reported that the amount of tannins in the seeds of Syrah grapes varied between $1360-2830 \mathrm{mg} \mathrm{kg}^{-1}$ and approximately half of the tannins in wines passed from the seeds.

\section{Conclusion}

In conclusion, in this study conducted with 29 different grape varieties, it was determined that grape varieties are rich in phenolic compounds and their content significantly differs from each other. Comparing the white and colored varieties; it has been found that colored varieties had higher amount of total phenolic compound and anthocyanin content in skin, fruit flesh and seeds compared to the white varieties. Also, colored varieties had higher amount of tannin content in skin and fruit flesh than white varieties while it was the same in the seeds.

\section{Acknowledgments}

This study was produced from Aslı Polat's $\mathrm{PhD}$ thesis and it was supported by TAGEM (TAGEM/BBAD/14/A08/P04/08).

\section{Conflicts of Interest}

There is no conflict of interest between the authors.

\section{Author Contribution}

A.P. Performed the experiments, field work, took role in conceptualization, data analysis and writing of the manuscript. I.R. performed experiments and field work and participated in writing the manuscript. S.G. took role in conceptualization and writing the manuscript. All authors read and approved the final manuscript.

\section{References}

Acar, J. (1998). Phenolic Compounds and Natural Colors, Food chemistry, Ed. Saldaml, F. Hacettepe Univ. Publications, Ankara, p 435-452.

AOAC, (1998). Official Method of Analysis. $15^{\text {th }}$ Edition, Association of Official Analytical Chemists, Washington DC.

Aras, Ö. (2006). Determination of Total Carbohydrate, Protein, Mineral Substance and Phenolic Compound Content of Grapes and Grape Products. Süleyman Demirel University, Institute of Natural and Applied Sciences, Master Thesis, Isparta, p 59.

Bruno, G., \& Sparapano, L. (2007). Effects of three esca-associated fungi on Vitis vinifera L: V. Changes in the chemical and biological profile of xylem sap from diseased cv. Sangiovese vines. Physiological and Molecular Plant Pathology, 71, 210-229. http://dx.doi.org/10.1016/j.pmpp.2008.02.005

Camire, M. E., Chaovanalikit, A., Dougherty, M. P., \& Briggs, J. (2002). Blueberry and grape anthocyanins as breakfast cereal colorants. Journal of Food Science, 67(1), 438-441. https://doi.org/10.1111/j.13652621.2002.tb11425.x

Cangi, R., Saraçoğlu, O., Uluocak, E., Kiliç, D., \& Şen, A. (2011). Chemical changes during ripening in some wine grape varieties cultivated in Kazova (Tokat) Rregion. Journal of the Institute of Science and Technology, 1(3), 9-14.

Cemeroğlu, B., Yemenicioğlu, A., \& Özkan, M. (2001). Meyve ve Sebze Işleme Teknolojisi, Cilt 1, Meyve ve Sebzelerin Bileşimi Soğukta Depolanmaları. Gıda Teknolojisi Derneği Yayınları, Ankara, 24, p 328.

Costa, C. T., Horton, D., \& Margolis, S. A. (2000). Analysis of anthocyanins in foods by liquid chromatography, liquid chromatography-mass spectromery and capillary electrophoresis. Journal of Chromatography $A$, 881, 403-410. https://doi.org/10.1016/s0021-9673(00)00328-9

Dávalos, A., Bartolomé, B., \& Cordovés, C.G. (2005). Antioxidant properties of commercial grape juices and vinegars. Food Chemistry, 93(2), 325-330. http://dx.doi.org/10.1016/j.foodchem.2004.09.030 
Di Stefano, R., \& Cravero M. C. (1991). Metodi Per lo Studio dei Polifenoli dell'Uva. Rivista di Viticoltura e di Enologia 44(2), 37- 45.

Doshi, P., Adsule, P., \& Banerjee, K. (2006). Phenolic composition and antioxidant activity in grapevine parts and berries (Vitis vinifera L.) cv. Kishmish Chornyi (Sharad Seedless) during maturation. International Journal of Food Science and Technology, 41(1), 1-9. http://dx.doi.org/10.1111/j.1365-2621.2006.01214.x

Fernández-López, J.A., Hidalgo, V., Almela, L., \& Roca, J. M. L. (1992). Quantitatif changes in anthocyanins pigments of Vitis vinifera cv. Monastrell during maturation. Journal of the Science of Food and Agriculture, 58(1), 153-155. http://dx.doi.org/10.1002/jsfa.2740580127

Gagne, S., Saucier, C. \& Geny, L. (2006). Composition and cellular localization of tannins in Cabernet Sauvignon skins during growth. Journal of Agricultural and Food Chemistry, 54(25), 9465-9471. https://doi.org/10.1021/jf061946g

Gürsöz, S. (1993). GAP Alanına Giren Güneydoğu Anadolu Bölgesi Bağcılığı ve Özellikle Şanlıurfa İlinde Yetiştirilen Üzüm Çeşitlerinin Ampelografik Nitelikleri ile Verim ve Kalite Unsurlarının Belirlenmesi Üzerinde Bir Araştırma. Çukurova University, Institute of Natural and Applied Sciences, Doctorate Thesis, Adana, $p$ 363.

Harbertson, J. F., Kennedy, J. A., \& Adams, D. O. (2002). Tannin in skins and seeds of Cabernet Sauvignon, Syrah, and Pinot Noir berries during ripening. American Journal of Enology and Viticulture, 53(1):54-59.

Harborne, J. B., \& Grayer, R. J. (1993). Flavonoids and Insects. In The Flavonoids: Advances in Research Since 1986. J.B. Harborne (Ed.), Chapman \& Hall, London, p. 589-618.

Ho, P., Silvia, M. C., \& Hogg, T. A. (2001). Changes in colour and phenolic composition during the early stages of maturation of port in wood, stainless steel and glass. Journal of the Science of Food and Agriculture, 81(13), 1269-1280. https://doi.org/10.1002/jsfa.938

Jin, Z. M., He, J. J., Bi, H. Q., Cui, X.Y., \& Duan, C. Q. (2009). Phenolic compound profiles in berrys from nine red wine grape cultivars in Northwest China. Molecules, 14(12), 4922-4935. https://dx.doi.org/10.3390\%2Fmolecules14124922

Kaur, C. \& Kapoor, H. C. (2001). Antioxidants in fruits andv - the millennium's health. International Journal of Food Science+Technology, 36(7), 703-725. https://doi.org/10.1111/j.1365-2621.2001.00513.x

Kunter, B., Cantürk, S., \& Keskin, N. (2013). Histochemical Structure of Grape Berry. Journal of the Institute of Science and Technology 3(2), 17-24.

Makris, D. P., Boskou, G., Andrikopoulos, N. K., \& Kefalas, P. (2008). Characterisation of certain major polyphenolic antioxidants in grape (Vitis vinifera cv. Roditis) stems by liquid chromatography-mass spectrometry. European Food Research and Technology, 226(5), 1075-1079. https://doi.org/s00217-007-0633-9

Naczk, M., \& Shahidi, F. (2004). Extraction and analysis of phenolics in food. Journal of chromatography A, 1054(12), 95-111. https://doi.org/10.1016/s0021-9673(04)01409-8

Pastrana-Bonilla, E., Akoh, C. C., Sellappan, S., \& Krewer, G. (2003). Phenolic content and antioxidant capacity of Muscadine grapes. Journal of Agricultural and Food Chemistry, 51(18), 5497-4503. https://doi.org/10.1021/jf030113c

Peng, Z., Hayasaka, Y., Iland, P. G., Sefton, M., Hoj, P., \& Waters, E. J. (2001). Quantitative analysis of polymeric procyanidins (tannins) from grape (Vitis vinifera) seeds by reverse phase high-performance liquid chromatography. Journal of Agricultural and Food Chemistry, 49(1), 26-31. https://doi.org/10.1021/jf000670o

Revilla, I., Luisa, M., \& González-Sanjozé, L. (2001). Evolution during the storage of red wines treated with pectolytic enzymes: New anthocyanin pigment formation. Journal of Wine Research, 12(3), 183-197. https://doi.org/10.1080/09571260120106820

Robards, K., Prenzler, P.D., Tucker, G., Swatsiteng, P., \& Glover, W. (1999). Phenolic compounds and their role in oxidative processes in fruits. Food Chemistry, 66(4), 401-436. https://doi.org/10.1016/S03088146(99)00093-X

Singleton, V. L., \& Rossi, J. R. (1965). Colorimetry of total phenolics with phosphomolibdic - phosphothungstic acid. American Journal of Enology and Viticulture, 16(3), 144-158.

Sivritepe, N. (2001). Oxidative stress in nature: antioxidants in grapevine, grape and wine (in Turkish with English abstract). Journal of Aegean Agriculture Research Institute, 11(2), 108-135.

Souquet, J. M., Labarbe, B., Le Guerneve, C., Cheyneir, V., \& Moutounet, M. (2000). Phenolics composition of grape stems. Journal of Agricultural and Food Chemistry, 48(4), 1076-1080. https://doi.org/10.1021/jf991171u

Tangolar, S., Büyüktaş, N., Gök, S., \& Ergenoğlu, F. (1999). Distribution of Phenolic Compounds in Vine Shoot Tips During Vegetation Period and Its Effect on Shoot Tip Culture (in Turkish with English abstract). III. National Horticultural Congress, Ankara, Turkey, 14 - 17 September, 383-388 p. 
Toprak, F. E. (2011). Studies on the Phytochemical Properties of Kalecik Karası Grape Varieties Cultivated in Ankara and Nevşehir Provinces. Ankara University, Institute of Natural and Applied Sciences, Master Thesis, Ankara. 64 p.

Torchio, F., Cagnasso, E., Gerbi, V., \& Rolle, L. (2010). Mechanical properties, phenolic composition and extractability indices of Barbera grapes of different soluble solids contents from several growing areas. Analytica Chimica Acta, 660(1-2), 183-189. https://doi.org/10.1016/j.aca.2009.10.017

Van Buren, J. (1970). Fruit Phenolics. The Biochemistry of Fruits and Their Products, Vol:1 (Ed: Hulme, A. C.), Academic Pres, London, p 269-304.

Yavaş, I., \& Fidan, Y. (1986). The Value of Grapes in Human Nutrition. The Problems of the Food Industry and the Expected Effect of the Free Zone on the Food Industry Symposium. 15-17 October, Adana, p 225-236.

Yayla, F. (2008). Investigation of Wine Characteristics of Grape Varieties in the National Collection Vineyard (in Turkish with English abstract, interim report 2006-2008). Tekirdağ Viticulture Research Institute. https://arastirma.tarimorman.gov.tr/bagcilik/Menu/7/Sonuclanan-Projeler. Acess: 15.06 .2021

Yu, D., \& Meredith, C. P. (1986). The influence of explant origin on tissue browning and shoot production in shoot tip cultures of grapevine. Journal of the American Society for Horticultural Science, 111(6), 972-975. 